Article

\title{
Phenethyl Isothiocyanate and Cisplatin Co-Encapsulated in a Liposomal Nanoparticle for Treatment of Non-Small Cell Lung Cancer
}

\author{
Mengwei Sun ${ }^{1}$, Yi Shi ${ }^{1}$, Utkarsh J. Dang ${ }^{2}$ and Anthony J. Di Pasqua ${ }^{1, *}$ \\ 1 Department of Pharmaceutical Sciences, School of Pharmacy and Pharmaceutical Sciences, \\ Binghamton University; Johnson City, NY 13790, USA; msun22@binghamton.edu (M.S.); \\ yishi@binghamton.edu (Y.S.) \\ 2 Department of Health Outcomes and Administrative Sciences, School of Pharmacy \\ and Pharmaceutical Sciences, Binghamton University; Johnson City, NY 13790, USA; \\ udang@binghamton.edu \\ * Correspondence: dipasqua@binghamton.edu; Tel.: +1-607-777-5815
}

Academic Editor: Ashok Kakkar

Received: 3 January 2019; Accepted: 19 February 2019; Published: 22 February 2019

\begin{abstract}
Lung cancer is the leading cause of cancer-related death in the Unites States, and approximately $85 \%$ of all lung cancers are classified as non-small cell lung cancer (NSCLC), which is extremely difficult to treat and its survival rate is low. After decades of clinical trials, the most effective treatments are still those that implement the first-generation platinum anticancer agent cisplatin (CDDP) in combination with other drugs. We previously demonstrated that the naturally-occurring compound phenethyl isothiocyanate (PEITC) can be used to sensitize NSCLC cells to CDDP. Furthermore, co-encapsulation of PEITC and CDDP in liposomes enhances their toxicity toward NSCLC cells. We here optimize liposomal-PEITC-CDDP, demonstrate the release of PEITC and CDDP from the nanoparticle, and show that liposomal-PEITC-CDDP is much more toxic toward both A549 and H596 human NSCLC cell lines than toward WI-38 and BEAS-2B human normal lung cell lines. Thus, we have prepared an efficacious therapy that has significantly higher toxicity toward cancer cell lines than normal cell lines.
\end{abstract}

Keywords: NSCLC; liposomes; cisplatin; isothiocyanate; chemotherapy; toxicity

\section{Introduction}

Lung cancer is the leading cause of cancer-related death in the United States [1]. Approximately 85\% of all lung cancers are classified as non-small cell lung cancer (NSCLC), which is extremely difficult to treat and its survival rate is low [1]. After decades of clinical trials, the most effective treatments are still those that implement the first-generation platinum anticancer agent cisplatin (CDDP) in combination with other drugs [2]. Tubulin-binding agents, such as paclitaxel, are often used in combination with CDDP for treatment [3]. Microtubules, which are assembled from dimers of $\alpha$-and $\beta$-tubulin, are critical for cell division, making tubulin proteins an important target in rapidly dividing NSCLC cells. Unfortunately, both CDDP and the agents used in combination with CDDP are toxic to the patient. Furthermore, tumors often gain resistance to CDDP. One of the major issues associated with CDDP is its nephrotoxicity, and when this is coupled with the neurotoxicity associated with the typically used tubulin binding agents, taxanes and vincas $[3,4]$, the patient experiences significant side-effects. Thus, a treatment modality that aggressively decreases tumor volume, but has less toxicity to the patient has been sought. It has been demonstrated that the liposomal CDDP formulation LipoPlatin ${ }^{\mathrm{TM}}$ is less toxic to patients than free CDDP is, but has a similar efficacy against NSCLC [5]. In a randomized clinical trial, the effectiveness of LipoPlatin ${ }^{\mathrm{TM}}$ with the $\beta \mathrm{x}$-tubulin binding agent paclitaxel was similar to that 
of CDDP with paclitaxel; however, much less toxicity to the patient was observed [6]. Not all tubulin binding agents are neurotoxic; indibulin, for example, was reported to have specificity for unmodified microtubules and, thus, no neurotoxicity in preclinical studies [7].

In 2010, we showed that naturally occurring isothiocyanates (ITCs) can be used to enhance the efficacy of CDDP [8]; ITCs are metabolites of glucosinolates which are contained in cruciferous vegetables such as watercress [9]. In the aforementioned study, structural variations among the naturally-occurring ITCs affected their ability to sensitize NSCLC cells to CDDP, and this correlated well with their ability to bind to and, ultimately, degrade $\beta$-tubulin in NSCLC; the ability of benzyl ITC (BITC) is about the same as that of phenethyl ITC (PEITC), both of which are much greater than that of sulforaphane (SFN) [10]. Thus, this mechanism may be important for said sensitization. This mechanism was further elucidated in a study demonstrating that allyl isothiocyanate enhances CDDP toxicity against lung and ovarian cancer cells [11].

In a separate report, a synthetic ITC derivative, ethyl 4-isothiocyantobutanoate, was shown to sensitize ovarian cancer cells to CDDP and, moreover, it allowed for CDDP to overcome CDDP-resistance in cell culture [12]. Then, it was shown that naturally-occurring ITCs sensitize cervical and breast cancers to CDDP, but not human normal mammary epithelial cells [13], and, in another study, the naturally-occurring ITC BITC sensitized leukemia cells to CDDP, but not normal human lymphocytes [14]. These results, combined with the observation that micromolar plasma concentrations of ITCs are safely maintained in humans [15], suggest that using ITCs with CDDP may be an efficacious treatment modality with fewer side effects than those currently in use.

In 2014, we reported on the preparation of a liposomal nanoparticle containing both PEITC and CDDP [16]. Studies had shown that drugs reformulated in liposomes have an increased circulation time in the bloodstream and, furthermore, increased accumulation in tumors, which is due to the enhanced permeability and retention (EPR) effect [17]. Liposomes containing the relatively hydrophilic CDDP and hydrophobic PEITC were prepared and characterized. For this preparation, 1,2-distearoyl-sn-glycero-3-phosphocholine (DSPC) was chosen. Liposomes composed of DSPC were previously shown to have greater drug retention over $48 \mathrm{~h}$ at 4 and $37^{\circ} \mathrm{C}$ than those composed of phospholipids with lower phase transition temperature values [18]. These nanoparticles were uniform, with a size of approximately $140 \mathrm{~nm}$, and had a zeta potential of approximately $-65 \mathrm{mV}$, which indicates high stability of the formulation. The liposomal-PEITC-CDDP formulation was more toxic toward $\mathrm{H} 596$ cells than the combination co-administered as free drugs and liposomes containing only CDDP or PEITC.

We here expand on our previous work. Again, DSPC was used; however, the optimized liposomal nanoparticles have a 1:3 ratio of CDDP to PEITC, whereas the previously reported nanoparticles had a 1:2 ratio, so less CDDP can be administered. Careful characterization studies were performed and release profiles obtained for liposomal-CDDP, liposomal-PEITC and liposomes containing both CDDP and PEITC: liposomal-PEITC-CDDP. Then, toxicity was tested in two human NSCLC cell lines, A549 and H596, and compared to its toxicity in human normal lung cells, BEAS-2B and WI-38.

\section{Results and Discussion}

Blank and drug-encapsulated liposomes were prepared with the combination of DSPC:EPG in the ratio of 4:1. Various quantities of PEITC and/or CDDP were added to the liposomal formulation (Table 1). Percent loading and encapsulation efficiency (EE) of the two drugs increased with their concentrations, respectively (Table 1$)$. The highest loading $(1.35 \pm 0.27 \%)$ and EE $(83.9 \pm 4.1 \%)$ for CDDP in liposomes (Lipo-CDDP) was achieved using $8.33 \mu \mathrm{mol}$ of CDDP; the highest loading $(3.66 \pm 0.35 \%)$ and EE $(37.0 \pm 2.4 \%)$ for PEITC in liposomes (Lipo-PEITC) was achieved using $134 \mu \mathrm{mol}$ of PEITC. In the PEITC and CDDP-loaded liposomal formulation (Lipo-PEITC-CDDP), $8.33 \mu \mathrm{mol}$ of CDDP and $134 \mu \mathrm{mol}$ of PEITC were used to obtain $1.37 \pm 0.18 \%$ and $3.24 \pm 0.47 \%$ loading of CDDP and PEITC, respectively. 
Table 1. Percent drug loading and encapsulation efficiency (EE) of cisplatin (CDDP) and/or phenethyl isothiocyanate (PEITC) in liposomes.

\begin{tabular}{cccccccc}
\hline $\begin{array}{c}\text { DSPC } \\
(\mu \mathrm{mol})\end{array}$ & $\begin{array}{c}\text { EPG } \\
(\mu \mathrm{mol})\end{array}$ & $\begin{array}{c}\text { CDDP } \\
(\mu \mathrm{mol})\end{array}$ & $\begin{array}{c}\text { PEITC } \\
(\mu \mathrm{mol})\end{array}$ & $\begin{array}{c}\text { CDDP- } \\
\text { Loading }(\%)\end{array}$ & $\begin{array}{c}\text { CDDP- EE } \\
(\%)\end{array}$ & $\begin{array}{c}\text { PEITC- } \\
\text { Loading }(\%)\end{array}$ & $\begin{array}{c}\text { PEITC- EE } \\
(\%)\end{array}$ \\
\hline 16 & 4 & 4 & 0 & $0.56 \pm 0.29$ & $60.5 \pm 6.7$ & - & - \\
16 & 4 & 6 & 0 & $0.67 \pm 0.33$ & $79.8 \pm 5.2$ & - & - \\
$16 *$ & 4 & 8.33 & 0 & $1.35 \pm 0.27$ & $83.9 \pm 4.1$ & - & - \\
16 & 4 & 0 & 68 & - & - & $2.05 \pm 0.21$ & $26.7 \pm 3.1$ \\
16 & 4 & 0 & 108.8 & - & - & $2.89 \pm 0.32$ & $31.5 \pm 4.7$ \\
$16 *$ & 4 & 0 & 134 & - & - & $3.66 \pm 0.35$ & $37.0 \pm 2.4$ \\
$16^{*}$ & 4 & 8.33 & 134 & $1.37 \pm 0.18$ & $84.3 \pm 2.6$ & $3.24 \pm 0.47$ & $34.7 \pm 3.2$ \\
\hline
\end{tabular}

* Formulations that have the highest drug loadings, respectively, were chosen to be tested in cell studies.

Particle size distributions and zeta potentials of blank and PEITC and/or CDDP loaded liposomes were characterized using a Zetasizer. As shown in Table 2, the average diameter of these liposomes ranged from 116.3 (blank liposomes) to $173.4 \mathrm{~nm}$ (Lipo-PEITC-CDDP). The average polydispersity indexes (PDI) of these nanoparticles indicate uniform particle size and good dispersion. The stability of the blank liposomes and drug-encapsulated liposomes was verified by measuring their zeta potentials, which ranged from -40 to $-60 \mathrm{mV}$. It is shown in Figure $1 \mathrm{~A}$ that Lipo-PEITC-CDDP has a narrow size distribution, which indicates uniformity. Field Emission Scanning Electron Microscope (FESEM) was used to characterize the morphology of Lipo-PEITC-CDDP. SEM observation confirmed that the Lipo-PEITC-CDDP exhibits spherical morphology, with an average particle size of approximately $150 \mathrm{~nm}$.

Table 2. Size and zeta potentials of blank liposomes and liposomes loaded with cisplatin (CDDP) and/or phenethyl isothiocyanate (PEITC).

\begin{tabular}{|c|c|c|c|c|c|c|}
\hline $\begin{array}{c}9 \\
(\mu \mathrm{mol})\end{array}$ & $\begin{array}{c}\text { EPG } \\
(\mu \mathrm{mol})\end{array}$ & $\begin{array}{l}\text { CDDP } \\
(\mu \mathrm{mol})\end{array}$ & $\begin{array}{l}\text { PEITC } \\
(\mu \mathrm{mol})\end{array}$ & Size (nm) & $\begin{array}{l}\text { Polydispersity } \\
\text { Index (PDI) }\end{array}$ & Zeta $(\mathrm{mV})$ \\
\hline 16 & 4 & 0 & 0 & $116.3 \pm 15.2$ & 0.06 & $-41.0 \pm 5.2$ \\
\hline 16 & 4 & 4 & 0 & $142.4 \pm 26.4$ & 0.08 & $-45.3 \pm 7.4$ \\
\hline 16 & 4 & 6 & 0 & $149.2 \pm 36.7$ & 0.08 & $-46.3 \pm 7.2$ \\
\hline $16^{*}$ & 4 & 8.33 & 0 & $155.6 \pm 29.5$ & 0.09 & $-51.2 \pm 6.5$ \\
\hline 16 & 4 & 0 & 68 & $139.2 \pm 35.1$ & 0.12 & $-50.6 \pm 4.1$ \\
\hline 16 & 4 & 0 & 108.8 & $157.5 \pm 28.3$ & 0.13 & $-52.4 \pm 7.8$ \\
\hline $16^{*}$ & 4 & 0 & 134 & $165.6 \pm 20.2$ & 0.16 & $-54.5 \pm 6.3$ \\
\hline $16^{*}$ & 4 & 8.33 & 134 & $173.4 \pm 26.8$ & 0.22 & $-61.8 \pm 6.9$ \\
\hline
\end{tabular}

* Formulations that have the highest drug loadings, respectively, were chosen to be tested in cell studies.

(A)

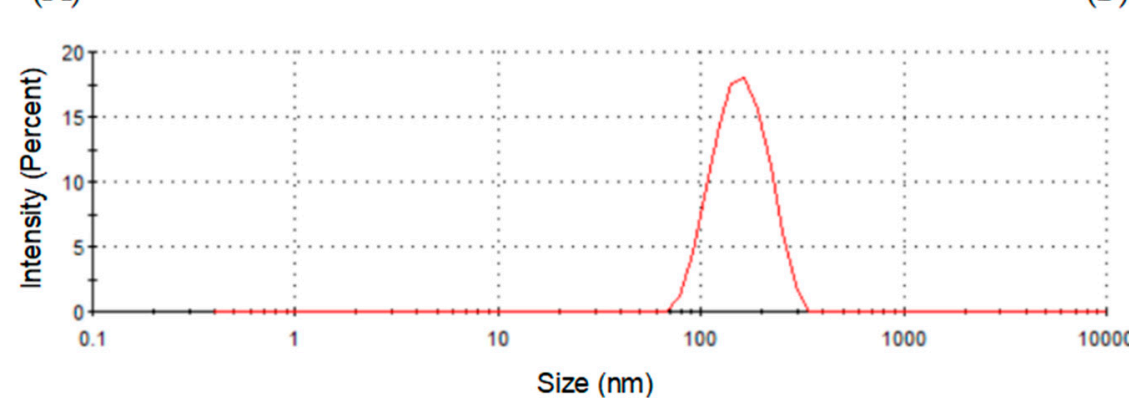

(B)

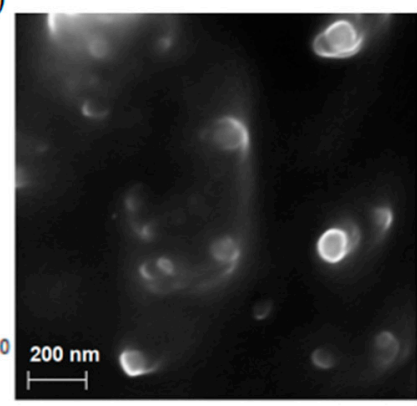

Figure 1. (A) Size distribution image of liposomes containing both phenethyl isothiocyanate (PEITC) and cisplatin (CDDP) (Lipo-PEITC-CDDP) and (B) SEM image of Lipo-PEITC-CDDP.

Drug release of nanoparticles is an important factor in determining the biological efficiency of drug delivery vehicles. Plots of percentage of CDDP and/or PEITC released from liposome formulations versus 
time are shown in Figure 2. The amount of CDDP released from Lipo-CDDP and Lipo-PEITC-CDDP over $24 \mathrm{~h}$ was measured using inductively-coupled plasma-mass spectrometry (ICP-MS). As is shown in Figure 2A and 2C, Lipo-CDDP and Lipo-PEITC-CDDP release $76.5 \pm 3.9 \%$ and $75.2 \pm 3.3 \%$ of their CDDP, respectively, in $2 \mathrm{~h}$. Over $24 \mathrm{~h}$, the amounts of CDDP released reached $86.1 \pm 4.3 \%$ and $91.1 \pm 2.9 \%$ for Lipo-CDDP and Lipo-PEITC-CDDP, respectively. A 1,2-benzenedithiol (BDT) assay was used to obtain the PEITC release profile of Lipo-PEITC and Lipo-PEITC-CDDP [19]. As is shown in Figure 2B, Lipo-PEITC showed a relatively sustained PEITC release, with $40.2 \pm 2.5 \%$ PEITC released gradually from Lipo-PEITC in the first $8 \mathrm{~h}$. The percent release remained almost the same up to $24 \mathrm{~h}$. A similar PEITC release profile was observed in Lipo-PEITC-CDDP (Figure 2C) with $44.8 \pm 2.6 \%$ of the PEITC released gradually within $8 \mathrm{~h}$. The release rate of CDDP in Lipo-PEITC-CDDP was higher than that of PEITC.

The MTS (3-(4,5-dimethylthiazol-2-yl)-5-(3-carboxymethoxyphenyl)-2-(4-sulfophenyl)-2H -tetrazolium) assay can be used to measure the change in cell viability caused by a change in metabolic activities. The cytotoxicity of free PEITC and/or CDDP, blank liposomes, drug-loaded liposomes toward human NSCLC cells (H596 and A549) and normal lung cells (WI-38 and BEAS-2B) (Figures 3 and 4 ) were tested and compared using the MTS assay. All four cell lines were treated and incubated for $24 \mathrm{~h}$. Figures 3 and 4 show plots of the toxicity of various drugs, drug combinations and liposome formulations toward cells. The cytotoxicities of the various treatments were compared based on the average and standard deviation of each group $(n=6)$, which are shown as error bars in Figures 3 and 4. 


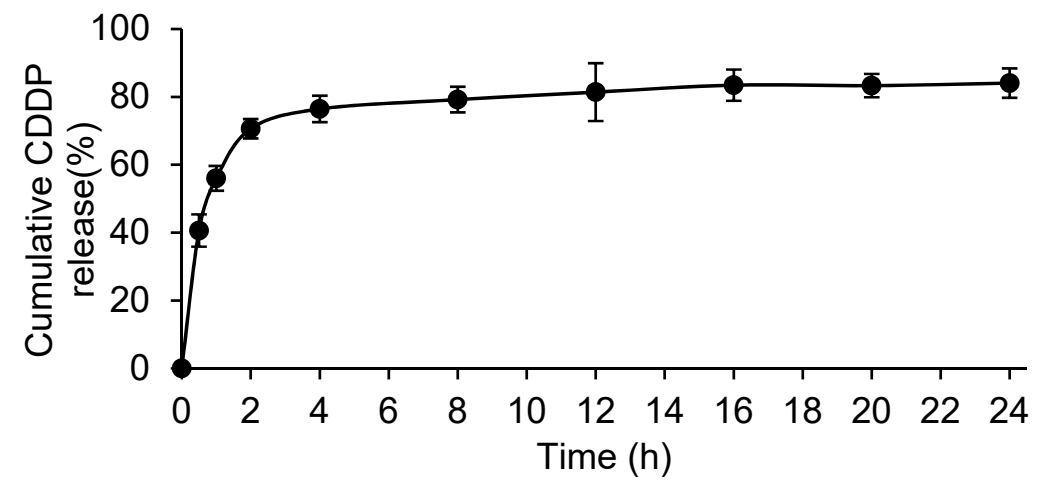

(A)

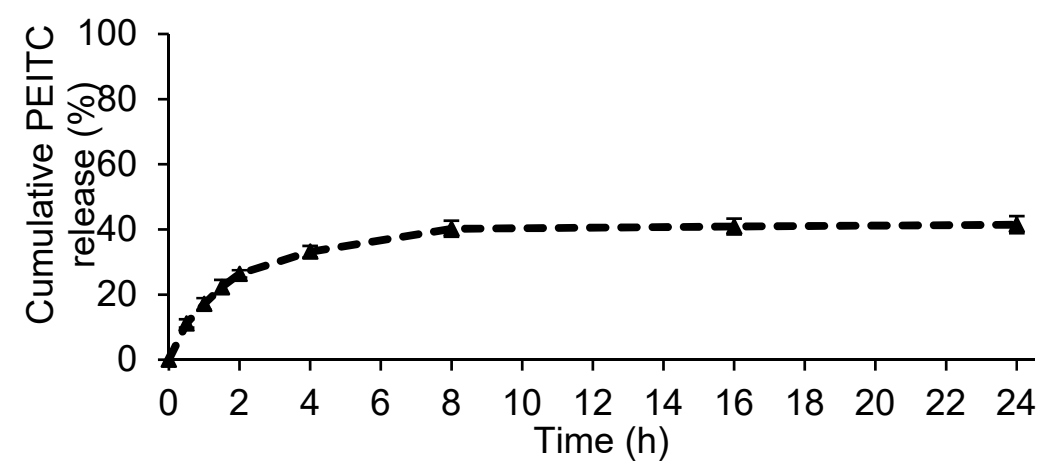

(B)

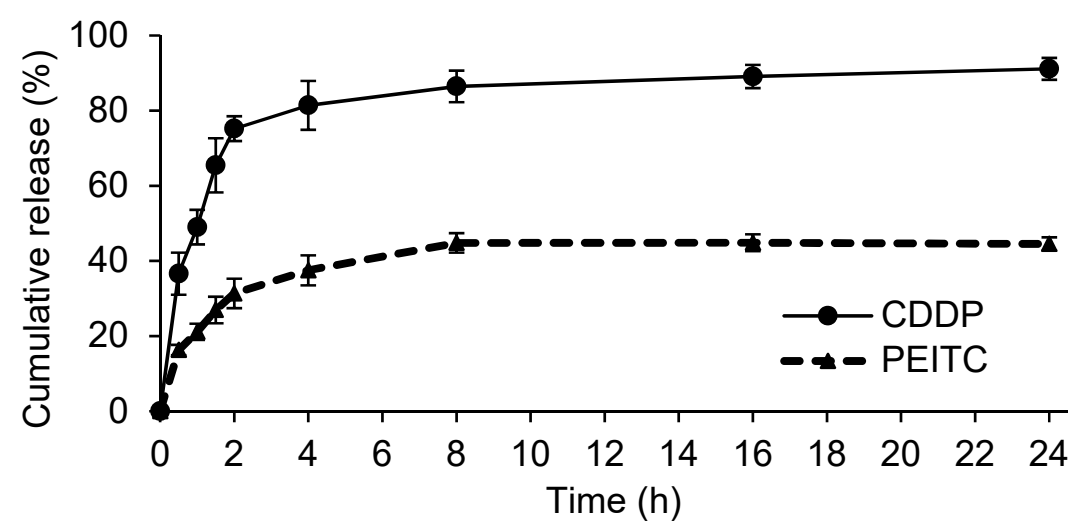

(C)

Figure 2. (A) In vitro cisplatin (CDDP) release profile from liposomes containing CDDP (Lipo-CDDP); (B) in vitro phenethyl isothiocyanate (PEITC) release profile from liposomes containing PEITC (Lipo-PEITC); and (C) in vitro CDDP and PEITC release profiles from liposomes containing both compounds (Lipo-PEITC-CDDP).

\subsection{Effect of CDDP and PEITC Combination Therapy}

In the cytotoxicity study using A549 cells (Figure 3A), the percent cell survival when treated with CDDP $(5 \mu \mathrm{M})$ alone was $55.9 \pm 3.4 \%$, while treatment with PEITC $(15 \mu \mathrm{M})$ alone resulted in a percent cell survival of $79.2 \pm 3.8 \%$. When treated with a combination of $5 \mu \mathrm{M}$ CDDP and $15 \mu \mathrm{M}$ PEITC (CDDP + PEITC), A549 cells had a percent survival of $46.2 \pm 2.7 \%$. The cytotoxicity associated 
with the combination of CDDP and PEITC was greater than those associated with CDDP or PEITC alone. As is shown in Figure 3B, the viability of $\mathrm{H} 596$ cells after treatment was similar to that of A549 cells. The percent survival after treatment with CDDP $(5 \mu \mathrm{M})$ alone was $74.6 \pm 9.2 \%$, and the percent survival after treatment with PEITC $(15 \mu \mathrm{M})$ alone was $84.9 \pm 8.9 \%$. Cells treated with a combination of $5 \mu \mathrm{M}$ CDDP and $15 \mu \mathrm{M}$ PEITC had a percent survival of $55.0 \pm 9.5 \%$. There are significant differences among groups CDDP + PEITC, CDDP and PEITC, as determined using a one-way ANOVA in R, for both $\mathrm{A} 549\left(p=1.1 \times 10^{-14}\right)$ and $\mathrm{H} 596$ cell lines $\left(p=1.55 \times 10^{-7}\right)$ [20]. A Dunnett's post hoc test accounting for multiple comparisons shows that for both cancer cell lines, the percent survival for the cells treated with a combination of CDDP + PEITC is significantly lower than free drug CDDP and PEITC, respectively (Table 3). The significant difference between the percent survival of CDDP + PEITC treatment groups and free CDDP treatment groups in both A549 and H596 cells confirmed the reported sensitization role of PEITC [8].

Table 3. Effect of cisplatin (CDDP) and phenethyl isothiocyanate (PEITC) combination therapy.

\begin{tabular}{ccc}
\hline Cell Line & Comparison & $p$-Value * \\
\hline \multirow{2}{*}{ A549 } & CDDP + PEITC vs. CDDP & $2.4 \times 10^{-7}$ \\
& CDDP + PEITC vs. PEITC & $<1 \times 10^{-10}$ \\
H596 & CDDP + PEITC vs. CDDP & $1.49 \times 10^{-5}$ \\
& CDDP + PEITC vs. PEITC & $8.03 \times 10^{-8}$ \\
\hline * Results of statistical analysis conducted using R (R Core Team, 2018).
\end{tabular}

\subsection{Effect of Liposome}

The blank liposomes appeared to have no significant effect on cell growth with the percent survival of $92.6 \pm 3.7 \%, 90.7 \pm 9.1 \%, 85.5 \pm 19.9 \%$, and $96.0 \pm 14.2 \%$ for A549, H596, WI-38 and BEAS-2B, respectively. Liposomes containing CDDP $(5 \mu \mathrm{M})$ or PEITC $(15 \mu \mathrm{M})$ showed greater toxicities toward A549 than the free drugs. The percent cell survival after treatment with liposomes loaded with CDDP (Lipo-CDDP) was $43.4 \pm 4.0 \%$, and for liposomes with PEITC (Lipo-PEITC), the percent cell survival was $64.6 \pm 4.2 \%$. Similarly, in H596 cells, the percent survival of the Lipo-CDDP treatment group $(5 \mu \mathrm{M}$, $37.5 \pm 8.9 \%)$ was lower than that of the free CDDP group $(5 \mu \mathrm{M}, 74.6 \pm 9.2 \%)$, while cell percent survival of the Lipo-PEITC treatment group $(15 \mu \mathrm{M}, 69.8 \pm 9.8 \%)$ was also lower than free PEITC $(15 \mu \mathrm{M}$, $84.9 \pm 8.9 \%$ ). A Welch two-sample t-test found that the liposomal formulation of CDDP (Lipo-CDDP) exhibits a significantly higher cytotoxic effect than free CDDP on both A549 $\left(p=2.09 \times 10^{-5}\right)$ and H596 $\left(p=1.75 \times 10^{-6}\right)$ cell lines (Table 4$)$. Similarly, the liposomal formulation of PEITC (Lipo-PEITC) has a significantly higher cytotoxic effect than free PEITC on both A549 $\left(p=5.86 \times 10^{-6}\right)$ and H596 $\left(p=1.15 \times 10^{-3}\right)$ cell lines. Lipo-PEITC, Lipo-CDDP and Lipo-PEITC-CDDP groups in the two lung cancer lines showed significantly higher cytotoxicity effects than free PEITC, CDDP and CDDP + PEITC groups, respectively, which indicated that liposomes can be used as an effective drug delivery vehicle.

Table 4. Effect of liposome.

\begin{tabular}{ccc}
\hline Cell Line & Comparison & $p$-Value \\
\hline \multirow{2}{*}{ A549 } & Lipo-CDDP vs. CDDP & $2.09 \times 10^{-5}$ \\
& Lipo-PEITC vs. PEITC & $5.86 \times 10^{-6}$ \\
\multirow{2}{*}{ H596 } & Lipo-CDDP vs. CDDP & $1.75 \times 10^{-6}$ \\
& Lipo-PEITC vs. PEITC & $1.15 \times 10^{-3}$ \\
\hline
\end{tabular}

\subsection{Effect of Lipo-PEITC-CDDP on Cancer Cell Lines}

When treated with liposomes containing $5 \mu \mathrm{M}$ of CDDP and $15 \mu \mathrm{M}$ of PEITC (Lipo-PEITC-CDDP), the A549 cells had a percent survival of $33.3 \pm 2.9 \%$, which was greater than that of CDDP + PEITC, Lipo-CDDP and Lipo-PEITC. Like A549 cells, the lowest percent survival of H596 cells (28.6 $\pm 6.3 \%)$ 
occurred when treated by $5 \mu \mathrm{M}$ of CDDP and $15 \mu \mathrm{M}$ of PEITC loaded in liposomes (Lipo-PEITC-CDDP), which was lower than that of CDDP + PEITC, Lipo-CDDP and Lipo-PEITC. There is a significant difference among groups Lipo-PEITC-CDDP, CDDP + PEITC, Lipo-CDDP, and Lipo-PEITC as determined using a one-way ANOVA for both A549 $\left(p=1.26 \times 10^{-14}\right)$ and H596 cell lines $\left(p=5.58 \times 10^{-10}\right)$. A Dunnett's post hoc test accounting for multiple comparisons shows that for both cancer cell lines, the percent survival of the cells treated with Lipo-PEITC-CDDP is significantly lower than the free drug combination (CDDP + PEITC), as well as liposomal formulations of either single drug (Lipo-CDDP and Lipo-PEITC) (Table 5). Loaded with CDDP and PEITC, Lipo-PEITC-CDDP has the advantages of enhanced CDDP efficacy and effective liposomal drug delivery and showed the highest cytotoxicity against the two NSCLC cell lines.

Table 5. Effect of liposomes containing both phenethyl isothiocyanate (PEITC) and cisplatin (CDDP) (Lipo-PEITC-CDDP) on cancer cell lines.

\begin{tabular}{cc}
\hline \multicolumn{2}{c}{ A549 } \\
\hline Lipo-PEITC-CDDP vs. & $p$-Value \\
\hline CDDP + PEITC & $<1 \times 10^{-6}$ \\
Lipo-CDDP & $<1 \times 10^{-6}$ \\
Lipo-PEITC & $<1 \times 10^{-6}$ \\
\hline \multicolumn{2}{c}{ H596 } \\
\hline Lipo-PEITC-CDDP vs. & $p$-Value \\
\hline CDDP + PEITC & $<0.001$ \\
Lipo-CDDP & 0.0367 \\
Lipo-PEITC & $<0.001$ \\
\hline
\end{tabular}

A Welch two-sample t-test found that there is a significant difference $\left(p=1.16 \times 10^{-14}\right)$ between no treatment group and Lipo-PEITC-CDDP group for the A549 cell line. The t-value capturing the effect size of the difference is 68.0. Similarly, for H596, a difference between the two groups was found with a $t$-value of 23.9 and a $p$-value of $4.22 \times 10^{-8}$. On the other hand, the effect sizes of the difference on normal cell lines were much lower (WI-38: $t=4.23, p=5.10 \times 10^{-3}$; BEAS-2B: $t=4.72, p=4.07 \times 10^{-3}$ ) (Table 6). This result shows that the Lipo-PEITC-CDDP drug therapy leads to a more significant cytotoxicity toward cancer cell lines as compared with normal cell lines.

Table 6. Comparison of no treatment group with liposomes containing both phenethyl isothiocyanate (PEITC) and cisplatin (CDDP) (Lipo-PEITC-CDDP) on cancer and normal cell lines.

\begin{tabular}{ccc}
\hline Cell Line & $\boldsymbol{p}$-Value & $\boldsymbol{t}$-Value \\
\hline A549 & $1.16 \times 10^{-14}$ & 68.0 \\
H596 & $4.22 \times 10^{-8}$ & 23.9 \\
WI-38 & $5.10 \times 10^{-3}$ & 4.23 \\
BEAS-2B & $4.07 \times 10^{-3}$ & 4.72 \\
\hline
\end{tabular}




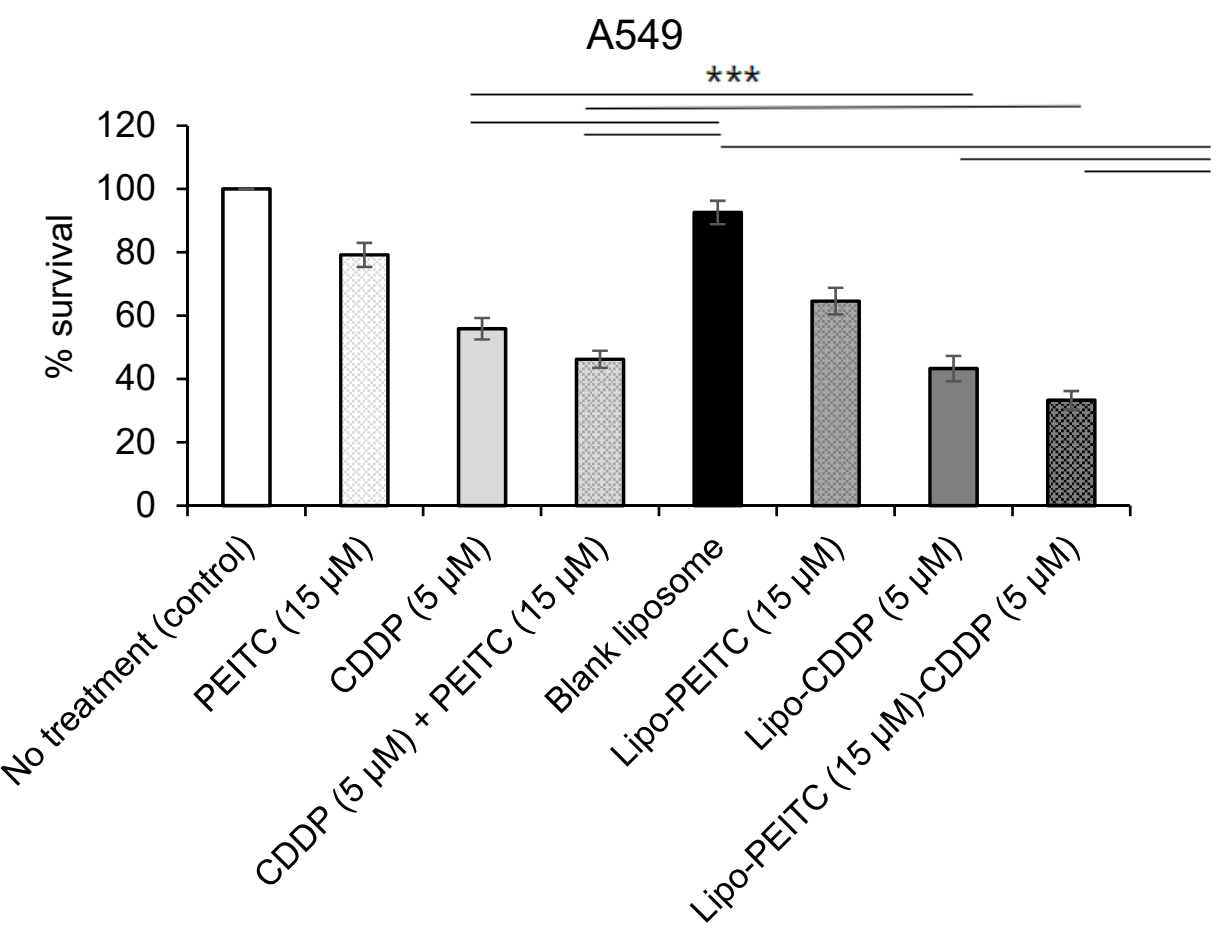

(A)

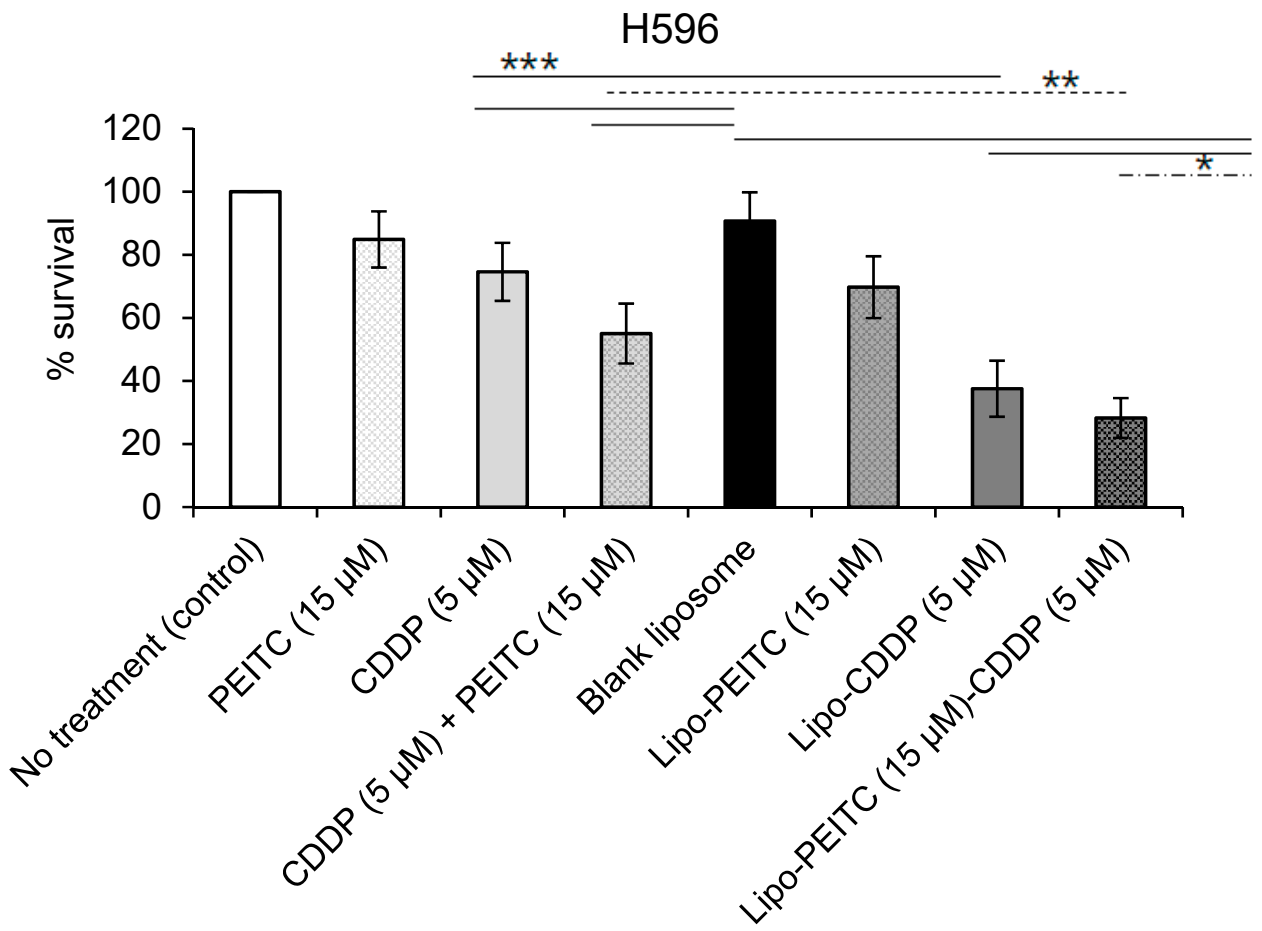

(B)

Figure 3. Percent survival of (A) human non-small cell lung cancer A549 and (B) H596 cells treated with free cisplatin (CDDP) and/or phenethyl isothiocyanate (PEITC), and liposome encapsulated compounds (solid line stands for ${ }^{* *} p<0.005$, dash line stands for ${ }^{* *} p<0.01$ and long dash dot line stands for $* p<0.05)$. 
WI-38

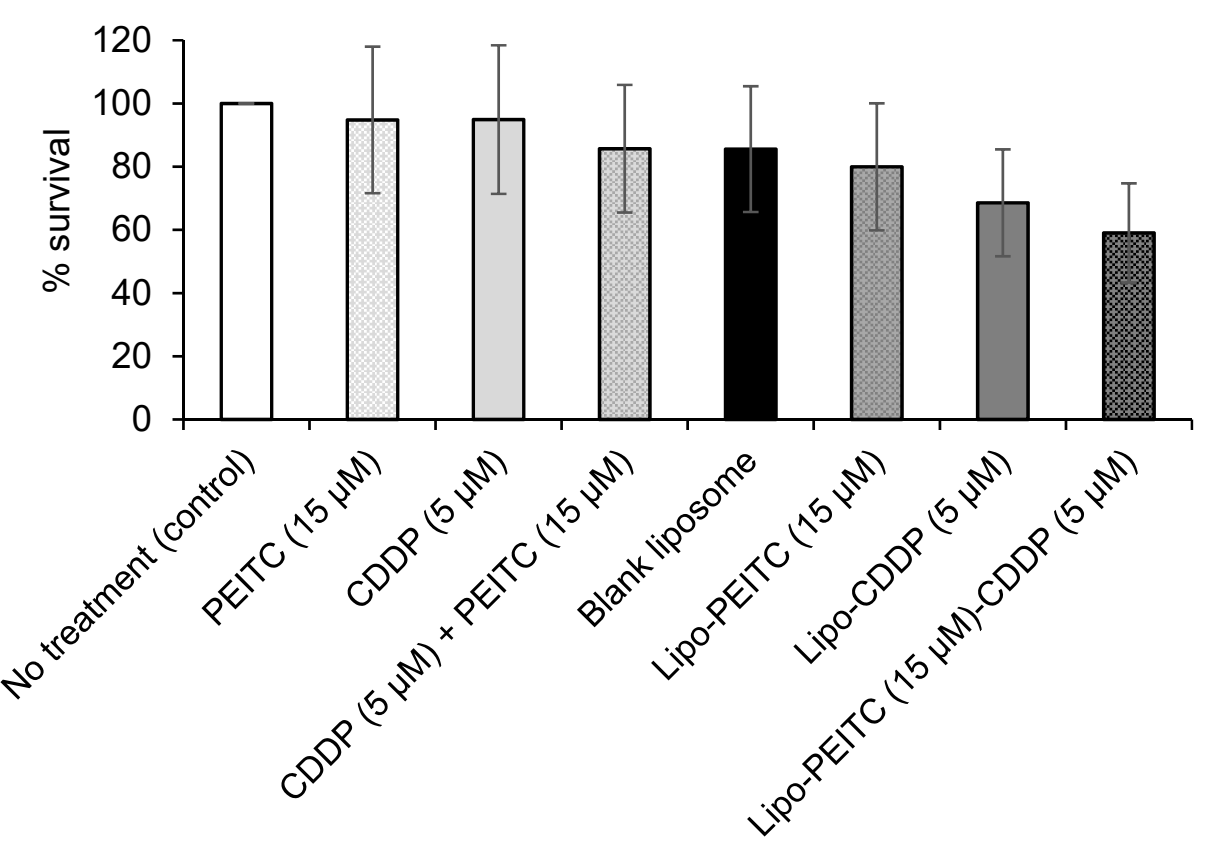

(A)

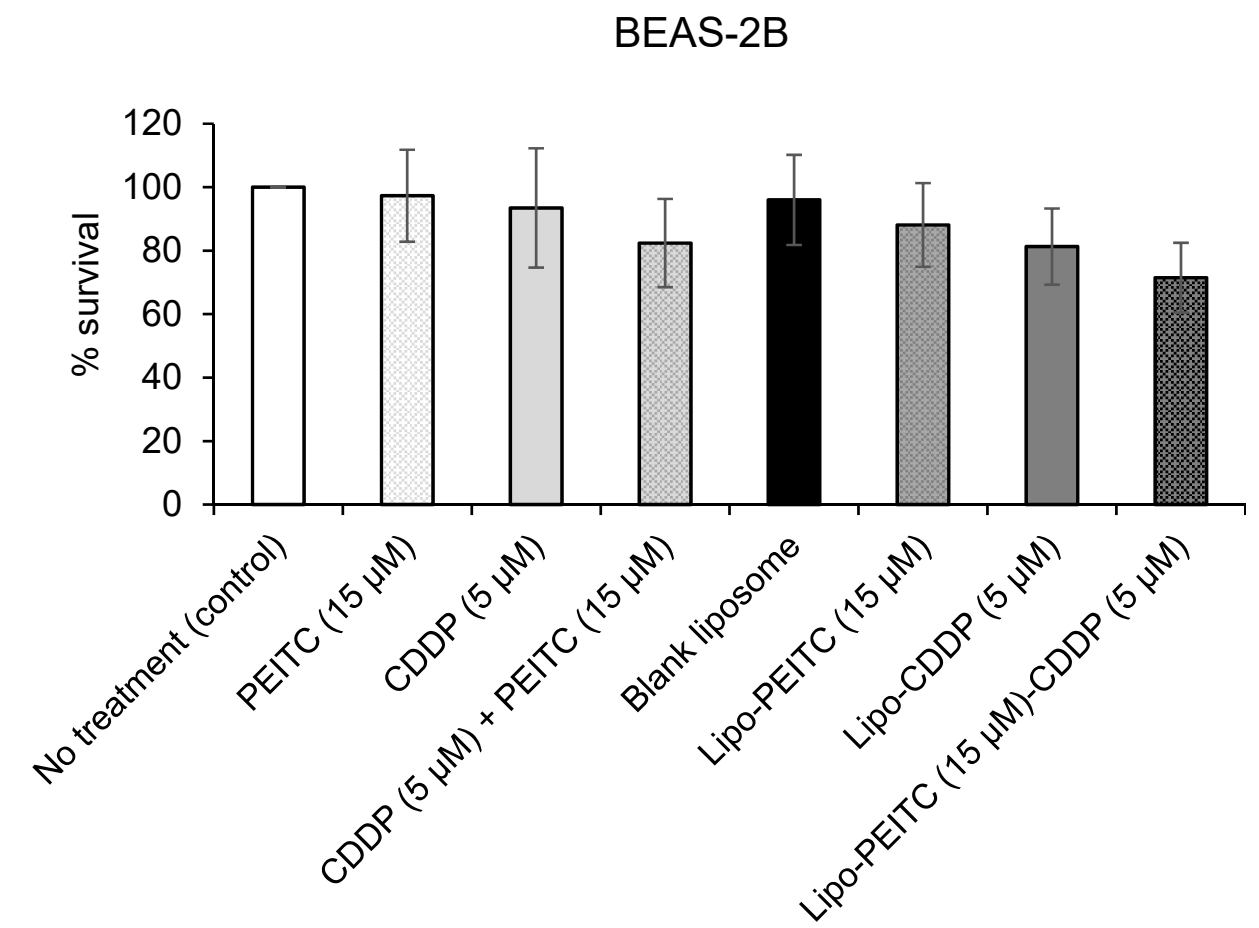

(B)

Figure 4. Percent survival of normal human lung cell lines (A) WI-38 (human diploid lung fibroblasts) and (B) BEAS-2B (human bronchial epithelial cells) treated with free cisplatin (CDDP) and phenethyl isothiocyanate (PEITC) and liposome-encapsulated compounds.

Because of our 3:1 PEITC:CDDP ratio, we were able to use less cisplatin in the Lipo-PEITC-CDDP against NSCLC cells than in our last formulation, which was 2:1 PEITC: CDDP [16]. We were able to 
achieve similar toxicity profiles using less CDDP and a higher concentration of PEITC. Cisplatin is a known nephrotoxic agent, so reducing its concentration to get a similar toxicity profile in NSCLC cells is advantageous. For the two normal lung cell lines (Figure 4A,B), while the Lipo-PEITC-CDDP caused the lowest survival percentages, $59.0 \pm 15.7 \%$ and $71.5 \pm 11.0 \%$ for WI-38 and BEAS-2B, respectively, the cytotoxic effect of Lipo-PEITC-CDDP was much greater in NSCLC cells. Therefore, the PEITC and CDDP combined liposomal therapy has a high therapeutic index.

\section{Materials and Methods}

\subsection{Materials}

1,2-distearoyl-sn-glycero-3-phosphocholine (DSPC) and L- $\alpha$-phosphatidylglycerol (egg, chicken) (EPG) were obtained from Avanti Polar Lipids, Inc. (Alabaster, AL, USA). Chloroform, phenethyl isothiocyanate (PEITC), cisplatin (CDDP) and dialysis sacks (12,000 Da) were purchased from Sigma-Aldrich (St. Louis, MO, USA). The 1,2-benzenedithiol (BDT) was obtained from Thermo Fisher Scientific (Waltham, MA, USA). Platinum $(1000 \pm 3 \mu \mathrm{g} / \mathrm{mL})$, lutetium $(1000 \mu \mathrm{g} / \mathrm{mL})$ and gold $(1000 \mu \mathrm{g} / \mathrm{mL})$ standards were purchased from High-Purity Standards (North Charleston, SC, USA). The normal lung fibroblast cell line WI-38, normal bronchial epithelial cell line BEAS-2B and NSCLC cell lines H596 and A549 were purchased from the American Type Culture Collection (Manassas, VA, USA). CellTiter $96^{\circledR}$ AQueous One Solution Cell Proliferation Assay (MTS) was from Promega (Madison, WI, USA).

\subsection{Preparation and Characterization of Liposomes Encapsulated with CDDP and/or PEITC}

First, $12.6 \mathrm{mg}$ DSPC and $4.1 \mathrm{mg}$ EPG were added to a round-bottom flask with 10, 16 or $20 \mu \mathrm{L}$ of PEITC and $0.4 \mathrm{~mL}$ of chloroform. After $30 \mathrm{~min}$ of rotary evaporation, the dry, thin lipid film formed at the bottom of the flask was dissolved with $1 \mathrm{~mL}$ of normal saline solution containing 4,6 or $8.33 \mu \mathrm{mol}$ of CDDP. Then the mixture was sonicated for $2 \mathrm{~min}$ with a water-type sonicator (Branson Ultrasonics Corporation, Danbury, CT, USA) and vortexed for $30 \mathrm{~s}$ at $65^{\circ} \mathrm{C}$ to obtain the resultant drug-encapsulated liposomes. Multilamellar liposomes were extruded 10 times through $200 \mathrm{~nm}$ and $100 \mathrm{~nm}$ polycarbonate membranes at $65^{\circ} \mathrm{C}$ using an extruder (Avanti Polar Lipid, Inc., Alabaster, AL, USA) to achieve small unilamellar liposomes. The free CDDP and PEITC were removed using $50 \mathrm{kDa}$ ultracentrifugation filter columns (Millipore Corporation, Billerica, MA, USA) at the speed of $3000 \times \mathrm{g}$ for $20 \mathrm{~min}$. A Zetasizer Nano (Malvern Instruments, Worcestershire, UK) was used to investigate the particle size distribution and zeta potential of the liposomes prepared using the dynamic light scattering technique. The structure and morphology of PEITC and CDDP co-encapsulated liposomes were characterized by Field Emission Scanning Electron Microscope (FESEM), Supra 55 VP (Zeiss, Oberkochen, Germany).

\subsection{Determination of PEITC Loading in Liposomal-PEITC and Liposomal-PEITC-CDDP}

The amount of PEITC loaded in the liposomes was determined using a 1,2-benzenedithiol (BDT) assay reported by Zhang with minor modifications [19]. To $1 \mathrm{~mL}$ of liposomal-PEITC suspended in potassium phosphate solution $(100 \mathrm{mM}, \mathrm{pH} 8.5)$ or $1 \mathrm{~mL}$ of liposomal-PEITC-CDDP suspended in the same, was added $1 \mathrm{~mL}$ of $4 \mathrm{mM}$ BDT solution in methanol. Then the $2 \mathrm{~mL}$ reaction solution was heated in a closed glass vial at $65^{\circ} \mathrm{C}$ for $2 \mathrm{~h}$, and its absorbance at $365 \mathrm{~nm}$ was measured using a UV-Vis spectrophotometer SpectraMax (Molecular Devices, Sunnyvale, CA, USA). A standard curve was established using PEITC $\left(\mathrm{R}^{2}=0.98\right)$ in the same manner, and concentrations of PEITC in samples were determined based on the standard curve.

The PEITC drug loading was calculated using the equation:

$$
\text { PEITC loading }(\%)=\frac{\text { Weight of PEITC in liposomes }}{\text { Total weight of lipids and PEITC }} \times 100 \%
$$




\subsection{Determination of CDDP Loading in Liposomal-CDDP and Liposomal-PEITC-CDDP}

Cisplatin loading was determined using inductively-coupled plasma-mass spectrometry (ICP-MS, PerkinElmer 350D, Waltham, MA, USA). Liposomal-CDDP and liposomal-PEITC-CDDP were digested with $70 \%$ nitric acid and heated at $70{ }^{\circ} \mathrm{C}$ overnight to evaporate. Then, the sample was re-dissolved in $70 \%$ nitric acid and diluted using deionized water, and the final samples for the ICP-MS test contained $2 \%$ nitric acid. Lutetium (20 ppb) was used as an internal standard.

The CDDP drug loading was calculated using the equation:

$$
\text { CDDP loading }(\%)=\frac{\text { Weight of CDDP in liposomes }}{\text { Total weight of lipids and CDDP }} \times 100 \% \text {. }
$$

\subsection{In Vitro Drug Release Studies}

Different amounts of PEITC or CDDP were used to prepare liposomal-PEITC or liposomal-CDDP. The formulation that achieved the highest loading (\%) of PEITC is referred to as "Lipo-PEITC", the highest loading (\%) of liposomal-CDDP is referred to as "Lipo-CDDP" and the liposomal formulation which combined the highest loading (\%) of both PEITC and CDDP is referred to as "Lipo-PEITC-CDDP". These three liposomal formulations were tested for their in vitro drug release and cytotoxicity. Drug release was studied using a dialysis method. Dialysis sacks were rinsed thoroughly using deionized water for $30 \mathrm{~min}$, then $1 \mathrm{~mL}$ of Lipo-PEITC, Lipo-CDDP or Lipo-PEITC-CDDP was placed in the dialysis sack. The sack was fully immersed in a capped glass vial containing $20 \mathrm{~mL}$ of $1 \times$ PBS with $25 \% v / v$ methanol. A spin bar was added in the vial to achieve $100 \mathrm{rpm}$ rotation speed. The temperature was set at $37^{\circ} \mathrm{C}$, and aliquots $(100 \mu \mathrm{L})$ of the release medium were withdrawn for analysis at different time points (up to $24 \mathrm{~h}$ ) and replaced with fresh medium. The absorbance of PEITC released from Lipo-PEITC and Lipo-PEITC-CDDP was measured using the same BDT assay as described above. The amount of CDDP released from Lipo-CDDP and Lipo-PEITC-CDDP was determined using ICP-MS.

The percentage of release of CDDP or PEITC at different time points was calculated using the equation:

$$
\text { Release }(\%)=\frac{\text { Cumulative amount released }}{\text { Total amount in liposomes }} \times 100 \%
$$

\subsection{Cytotoxicity Studies}

All cell studies were carried out in a humidified $37^{\circ} \mathrm{C}, 5 \% \mathrm{CO}_{2}$ (standard conditions) atmosphere incubator. For the two normal cell lines, the culture medium used for the WI-38 cells was the minimum essential medium (MEM) containing $100 \mu \mathrm{g} / \mathrm{mL}$ streptomycin, 10\% fetal bovine serum (FBS), $2.0 \mathrm{mM}$ L-glutamine and $100 \mathrm{IU} / \mathrm{mL}$ penicillin; the culture medium used for the BEAS-2B cells was bronchial epithelial basal medium (BEBM) with 10\% FBS and supplements. The culture medium used for the two cancer cell lines, H596 and A549 cells, was Roswell Park Memorial Institute (RPMI) medium containing $100 \mathrm{\mu g} / \mathrm{mL}$ streptomycin, $100 \mathrm{IU} / \mathrm{mL}$ penicillin, 10\% FBS, and $2.0 \mathrm{mM} \mathrm{L-glutamine.}$

Nine groups $(n=6)$ were included to test the cytotoxicity toward each cell line. Two control groups were included: group 1, control group with only medium; group 2, control group with non-treated cells. Group 3-9 were cells with treatment: group 3, free CDDP; group 4, free PEITC; group 5, CDDP and PEITC (CDDP + PEITC); group 6, blank liposomes; group 7, Lipo-CDDP; group 8, Lipo-PEITC; group 9, Lipo-PEITC-CDDP. The cells were seeded at $5 \times 10^{3}$ cells $/ \mathrm{mL}(100 \mu \mathrm{L} /$ well $)$ in 96-well plates and allowed to grow for $24 \mathrm{~h}$; after that, the medium was removed and replaced with $100 \mu \mathrm{L}$ of medium containing the treatment suspensions, which was removed after an exposure time of $24 \mathrm{~h}$ and replaced with $100 \mu \mathrm{L}$ of fresh medium. To each well, $20 \mu \mathrm{L}$ of MTS solution was added and incubated for $2 \mathrm{~h}$, 
and the UV-Vis absorbance was read at $490 \mathrm{~nm}$. The percent survival of cells treated was calculated using the following equation:

$$
\operatorname{Survival}(\%)=\frac{A_{t}-A_{m}}{A_{c}-A_{m}} \times 100 \%
$$

where $A_{t}$ is the absorbance of cells in treatment groups, $A_{m}$ is the absorbance of medium alone and $A_{c}$ is the absorbance of cells without treatment. All $p$ values were calculated using Microsoft Excel $t$-test function (Redmond, WA, USA).

\subsection{Statistical Analyses}

All statistical analyses were conducted using $R$ [20]. For the comparison of two group means, two-sample Welch's $t$-tests were conducted using the t.test() function. The Levene test was carried out to test for homogeneity of variances using leveneTest() from the car package [21]. For comparison of more than two group means, one-way ANOVAs were conducted using the aov() function with a post-hoc Dunnett test carried out using the glht() function from the multcomp package [22].

\section{Conclusions}

Cisplatin is commonly used against NSCLC in the clinic; however, the negative side-effects associated with this metal-based drug urgently need to be addressed. We encapsulated CDDP and PEITC in liposomes, as this strategy has been shown to increase the circulation time of encapsulated CDDP and reduce its side-effects [17]. Furthermore, co-encapsulation of PEITC and CDDP in liposomes enhances their cytotoxicity toward NSCLC cells [16]. Release of PEITC and CDDP was studied and the cytotoxicity of this formulation against human NSCLC and normal lung cells determined. Lipo-PEITC-CDDP was significantly more toxic toward both NSCLC cell lines than toward the normal lung cell lines tested. Thus, we have prepared an efficacious therapy that is more toxic toward cancer cells than toward normal cells.

Author Contributions: M.S. performed experiments, interpreted results and worked on this manuscript. Y.S. interpreted results and worked on this manuscript. U.J.D. performed statistical analysis. A.J.D.P. designed the study, oversaw experiments and worked on this manuscript.

Funding: This research received no external funding.

Acknowledgments: The authors wish to thank the Binghamton University School of Pharmacy and Pharmaceutical Sciences for their financial support of this work. We also thank Ositomiwa O. Osipitan, Katelyn Wells and Jennifer R. Amey for their help pertaining to this work.

Conflicts of Interest: Authors declare no conflict of interest pertaining to this work.

\section{Abbreviations}

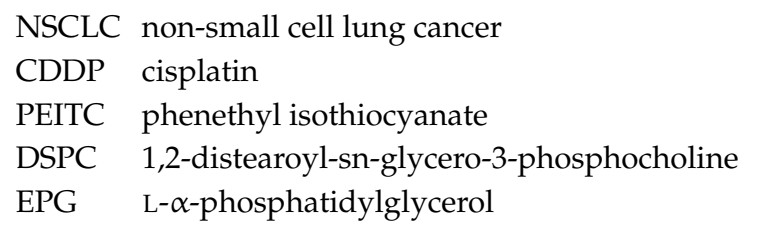

\section{References}

1. American Cancer Society. Cancer Facts and Figures 2018. Available online: https://www.cancer.org/research/ cancer-facts-statistics/all-cancer-facts-figures/cancer-facts-figures-2018.html (accessed on 12 October 2018).

2. Goffin, J.; Lacchetti, C.; Ellis, P.M.; Ung, Y.C.; Evans, W.K. First-line systemic chemotherapy in the treatment of advanced non-small cell lung cancer. J. Thorac. Oncol. 2010, 5, 260-274. [CrossRef] [PubMed]

3. Van den Bent, M.J. Prevention of chemotherapy-induced neuropathy: Leukemia inhibitory factor. Clin. Cancer Res. 2005, 11, 1691-1693. [CrossRef] [PubMed] 
4. Dumontet, C.; Jordan, M.A. Microtubule-binding agents: A dynamic field of cancer therapeutics. Nat. Rev. Drug Discov. 2010, 9, 790-803. [CrossRef] [PubMed]

5. Stathopoulos, G.P.; Antoniou, D.; Dimitroulis, J.; Stathopoulos, J.; Marosis, K.; Michalopoulou, P. Comparison of liposomal cisplatin versus cisplatin in non-squamous cell non-small-cell lung cancer. Cancer Chemother. Pharmacol. 2011, 68, 945-950. [CrossRef] [PubMed]

6. Stathopoulos, G.; Antoniou, D.; Dimitroulis, J.; Michalopoulou, P.; Bastas, A.; Marosis, K.; Stathopoulos, J.; Provata, A.; Yiamboudakis, P.; Veldekis, D.; et al. Liposomal cisplatin combined with paclitaxel versus cisplatin and paclitaxel in non-small cell lung cancer: a randomized phase III multicenter trial. Ann. Oncol. 2010, 21, 2227-2232. [CrossRef] [PubMed]

7. Wienecke, A.; Bacher, G. Indibulin, a novel microtubule inhibitor, discriminates between mature neuronal and nonneuronal tubulin. Cancer Res. 2010, 69, 171-177. [CrossRef] [PubMed]

8. Di Pasqua, A.J.; Hong, C.; Wu, M.Y.; McCracken, E.; Wang, X.; Mi, L.; Chung, F.-L. Sensitization of non-small cell lung cancer cells to cisplatin by naturally occurring isothiocyanates. Chem. Res. Toxicol. 2010, 23, 1307-1309. [CrossRef] [PubMed]

9. World Health Organization. IARC Handbook of Cancer Prevention. Available online: http://handbooks. iarc.fr/ (accessed on 12 October 2018).

10. Mi, L.; Xiao, Z.; Hood, B.L.; Dakshanamurthy, S.; Wang, X.; Govind, S.; Conrads, T.P.; Veenstra, T.D.; Chung, F.-L. Covalent binding to tubulin by isothiocyanates: A mechanism of cell growth arrest and apoptosis. J. Biol. Chem. 2008, 283, 22136-22146. [CrossRef] [PubMed]

11. Ling, X.; Westover, D.; Cao, F.; Cao, S.; He, X.; Kim, H.-R.; Zhang, Y.; Chan, D.; Li, F. Synergistic effect of allyl isothiocyanate (AITC) on cisplatin efficacy in vitro and in vivo. Am. J. Cancer Res. 2015, 5, 2516-2530. [PubMed]

12. Bodo, J.; Chovancova, J.; Hunakova, L.; Sedlak, J. Enhanced sensitivity of human ovarian carcinoma cell lines A2780 and A2780/CP to the combination of cisplatin and synthetic isothiocyanate ethyl 4-isothiocyanatobutanoate. Neoplasma 2005, 52, 510-516. [PubMed]

13. Wang, X.; Govind, S.; Sajankila, S.P.; Mi, L.; Roy, R.; Chung, F.-L. Phenethyl isothiocyanate sensitizes human cervical cancer cells to apoptosis induced by cisplatin. Mol. Nutr. Food Res. 2011, 55, 1-10. [CrossRef] [PubMed]

14. Lee, Y.; Kim, Y.J.; Choi, Y.J.; Lee, J.W.; Lee, S.; Chung, H.W. Enhancement of cisplatin cytotoxicity by benzyl isothiocyanate in HL-60 cells. Food Chem. Toxicol. 2012, 50, 2397-2406. [CrossRef] [PubMed]

15. Liebes, L.; Conaway, C. High-performance liquid chromatography-based determination of total isothiocyanate levels in human plasma: Application to studies with 2-phenethyl isothiocyanate. Anal. Biochem. 2001, 291, $279-289$. [CrossRef] [PubMed]

16. Yang, Y.-T.; Shi, Y.; Jay, M.; Di Pasqua, A.J. Enhanced toxicity of cisplatin with chemosensitizer phenethyl isothiocyanate toward non-small cell lung cancer cells when delivered in liposomal nanoparticles. Chem. Res. Toxicol. 2014, 27, 946-948. [CrossRef] [PubMed]

17. Al-Jamal, W.T.; Kostarelos, K. Liposomes: From a clinically established drug delivery system to a nanoparticle platform for theranostic nanomedicine. Acc. Chem. Res. 2011, 44, 1094-1104. [CrossRef] [PubMed]

18. Anderson, M.; Omri, A. The effect of different lipid components on the in vivo stability and release of liposome formulations. Drug Deliv. 2004, 11, 33-39. [CrossRef] [PubMed]

19. Zhang, Y. The 1,2-benzenedithiole-based cyclocondensation assay, a valuable tool for measurement of chemopreventive isothiocyanates. Crit. Rev. Food Sci. Nutr. 2012, 52, 525-532. [CrossRef] [PubMed]

20. R: A Language and Environment for Statistical Computing, version 3.5.2; R Foundation for Statistical Computing: Vienna, Austria, 2018.

21. Fox, J.; Weisberg, S. An R Companion to Applied Regression, 2nd ed.; Sage Publications: Thousand Oaks, CA, USA, 2011.

22. Hothorn, T.; Bretz, F.; Westfall, P. Simultaneous Inference in General Parametric Models. Biom. J. 2008, 50, 346-363. [CrossRef] [PubMed]

Sample Availability: Samples of the compounds and liposomes are available from the authors. 